\title{
Cutaneous manifestations in inflammatory bowel disease (Review)
}

\author{
LOREDANA UNGUREANU ${ }^{1}$, RODICA COSGAREA ${ }^{1}$, MIHAIL ALEXANDRU BADEA ${ }^{2}$, \\ ALINA FLORENTINA VASILOVICI ${ }^{1}$, IOANA COSGAREA ${ }^{3}$ and SIMONA CORINA ȘENILÄ ${ }^{1}$ \\ ${ }^{1}$ Department of Dermatology, Iuliu Hațieganu University of Medicine and Pharmacy, 400006 Cluj-Napoca; \\ ${ }^{2}$ Department of Dermatology, University of Medicine and Pharmacy of Targu Mures, 540139 Targu Mures, Romania; \\ ${ }^{3}$ Institute of Cellular Medicine, University of Newcastle, NE2 4HH Newcastle upon Tyne, UK
}

Received September 6, 2019; Accepted October 7, 2019

DOI: $10.3892 / \mathrm{etm} .2019 .8321$

\begin{abstract}
Inflammatory bowel disease (IBD) is defined as a chronic condition characterized by unpredictable relapsing episodes of gastrointestinal inflammation. IBD is not limited to the gastrointestinal tract and should be considered a systemic disease which can involve any organ. Cutaneous manifestations in IBD are frequent and comprise a broad spectrum of diseases, ranging from mild to severe and sometimes debilitating lesions. Some of the cutaneous manifestations can present signs of an underlying intestinal disease, leading to the screening for non-detected IBD even in the absence of symptoms. Cutaneous EIMs are divided into 4 categories: i) Disease-specific lesions that show the same histopathologic findings as the underlying gastrointestinal disease, ii) reactive lesions which are inflammatory lesions that share a common pathogenetic mechanism but do not share the same pathology with the gastrointestinal disease, iii) associated conditions are more frequently observed in the context of IBD, without sharing the pathogenetic mechanism or the histopathological findings with the underlying disease and iv) drug-related skin reactions.
\end{abstract}

\section{Contents}

1. Introduction

Correspondence to: Dr Rodica Cosgarea, Department of Dermatology, Iuliu Hațieganu University of Medicine and Pharmacy, 3-6 Clinicilor Street, 400006 Cluj-Napoca, Romania

E-mail: cosgarear@yahoo.com

Abbreviations: $\mathrm{CD}$, Crohn disease; CPN, cutaneous polyarteritis nodosa; EBA, epidermolysis bullosa acquisita; EN, erythema nodosum; EIM, extraintestinal manifestation; IBD, inflammatory bowel disease; NV, necrotizing vasculitis; PG, pyoderma gangrenosum; PV, pyostomatitis vegetans; SS, Sweet's syndrome; UC, ulcerative colitis

Key words: inflammatory bowel disease, extraintestinal manifestations, cutaneous manifestations, drug-related cutaneous manifestations, biologic therapy
2. Disease specific cutaneous manifestations

3. Reactive cutaneous manifestations

4. Associate cutaneous manifestations

5. Drug-related cutaneous manifestations

6. Conclusions

\section{Introduction}

Inflammatory bowel disease (IBD) is defined as a chronic condition characterized by unpredictable relapsing episodes of gastrointestinal inflammation. Classically, IBD is subdivided into Crohn disease (CD) and ulcerative colitis (UC), but the distinction among the two is sometimes difficult. Due to the lack of a single test for discrimination, $10 \%$ of IBD cases remaining unclassified (1). Although defined by the gastrointestinal inflammation, IBD is not limited to the gastrointestinal tract and should be considered a systemic disease which can involve any organ. Extraintestinal manifestations (EIMs) in IBD patients are defined as inflammatory entities caused by the same processes that drive inflammation in the gut, but which are located outside the intestine (2). The frequency of reported EIMs ranges from 6 to $47 \%$ (2) and interestingly EIMs can appear before or after the diagnosis of IBD is made. Vavricka et al (3) showed that in $25.8 \%$ of the cases, the first EIM occurred before IBD was diagnosed, with a median time of 5 months before the diagnosis. Although they can be located anywhere, EIMs most frequently affect joints, the skin, the hepatobiliary tract and the eye (2). It was shown that EIMs impact significantly the morbidity and mortality in patients with IBB $(4,5)$ and their presence should be a reason to screen for IBD in order not to delay the diagnosis and to promptly initiate therapy.

The skin and oral mucosa are easily accessible for examination and represent one of the important sites for EIMs. Cutaneous manifestation can be the presenting sign of IBD or can develop together with or after the gastrointestinal signs of the disease. They are described in up to $15 \%$ of the patients, although there are studies that report a higher rate (6). Cutaneous manifestations are more frequent in $\mathrm{CD}$, being reported in up to $43 \%$ of the patients $(6,7)$.

Classically, cutaneous manifestations in IBD were divided into 3 categories: i) disease-specific lesions that show the same histopathologic findings as the underlying gastrointestinal 
disease, ii) reactive lesions which are inflammatory lesions that share a common pathogenetic mechanism but do not share the same pathology with the gastrointestinal disease and iii) associated conditions are more frequently observed in the context of IBD, without sharing the pathogenetic mechanism or the histopathological findings with the underlying disease $(8,9)$. Due to the continuous development of therapeutic options for IBD and the risk of cutaneous adverse reactions associated with these treatments, a fourth category of cutaneous manifestations was proposed by some researchers, namely the drug-related cutaneous reactions.

Another classification of the cutaneous manifestations of IBD takes into account the correspondence between the course of the cutaneous disease and the one of the gastrointestinal disease. As a result, we have manifestations which have a parallel course with IBD, others which may or may not parallel IBD activity and finally manifestations with a separate course from IBD $(8,9)$.

The aim of the present review is to summarize the current knowledge on cutaneous manifestations in IBD.

\section{Disease specific cutaneous manifestations}

Disease specific manifestations are, as mentioned before, lesions that share the same histopathological findings, namely non-caseating granulomas, with IBD. Disease specific lesions are seen only in $\mathrm{CD}$, due to the fact that UC does not extend to external mucous membranes, being confined to the internal gastrointestinal tract (10).

Fissures and fistulae. There is controversy whether fissures and fistulae should be considered cutaneous EIMs or just an extension of the gastrointestinal disease. Perianal fissures and fistulae were observed in $36 \%$ of patients with CD and were absent in UC patients (11). It was shown that the presence of colitis is a strong positive predictor of perianal disease compared to patients with small bowel disease only. Chronic oedema and inflammation in fissures and fistulae, lead to the development of perianal cutaneous abscesses, acrochordons, and pseudo skin tags (12).

Oral Crohn's disease. The granulomatous process can extend into the oral cavity in $8-9 \%$ of patients with CD (12). Specific oral lesions include a cobblestone appearance of the oral mucosa; deep linear ulcerations; mucosal tags; swelling of the lips, cheeks and face; lip and tongue fissures; and mucogingivitis (13). Moreover, autoimmune changes of the minor salivary glands, and in consequence dry mouth were reported (13).

Metastatic Crohn's disease. Metastatic CD is an extension of the granulomatous pathology to sites which are not in continuity with the bowel. Although it can manifest anywhere, the metastatic lesions are predominantly located on the extremities and intertriginous areas; the face and genitalia are rarely affected (14,15). Metastatic CD presents as plaques, nodules, ulcerations, abscesses and fistulas $(8,12)$. Noteworthy, the severity of metastatic lesions is not correlated with the severity of underlying disease (16) and the surgical resection of the affected bowel segment does not guarantee resolving of the cutaneous lesions (9).

\section{Reactive cutaneous manifestations}

Reactive cutaneous manifestations are caused by the underlying IBD and do not exhibit similar pathologic features with the gastrointestinal disease, being present in both UC and CD. It is thought that a cross antigenicity between the skin and the intestinal mucosa is responsible for this type of reactions (17).

Erythema nodosum (EN). EN is the most common clinical form of septal panniculitis, the inflammation of subcutaneous fat tissue; and the most commonly described cutaneous manifestation of IBD occurring in $4-6 \%$ of patients $(12,18)$. EN is not exclusively associated with IBD, other triggers being infections, malignancies, drugs or even pregnancy (18). Studies show that EN has a higher incidence in CD than in UC and is seen more frequently in young, female patients $(9,12,18)$. EN appears usually in patients with known IBD, however, $15 \%$ of the cases may precede the diagnosis of IBD by up to five years $(12,18)$. Moreover, $\mathrm{EN}$ is frequently associated with eye and joint involvement, isolated colonic involvement, and pyoderma gangrenosum (PG) (19).

Clinically, EN is characterized by the rapid onset of symmetrical, tender, and red to violet subcutaneous nodules of $1-5 \mathrm{~cm}$ in diameter. The lesions are typically located on the extensor surfaces of the lower extremities, particularly the anterior tibial area; the face, trunk, neck and upper extremities are rarely involved. Cutaneous lesions of EN are associated frequently with systemic symptoms such as malaise, fatigue or arthralgia. Diagnosis can usually be made clinically, biopsy being rarely required. If performed, the histopathological exam reveals a septal panniculitis, while direct immunofluorescence reveals perivascular deposits of immunoglobulins and complement, suggesting an abnormal immunological response of common antigens between bowel bacteria and the skin (17). Although EN parallels underlying intestinal disease activity, being associated with IBD flares, severity of EN does not necessarily parallel severity of the IBD flares (20). In the majority of cases, EN is either self-limiting or the appropriate medical treatment of the underlying disease leads to the resolution of the lesions without scaring. Leg elevation, analgesics, potassium iodine, and compressive stockings are supportive treatment methods, while in severe cases, systemic corticosteroids may be required and represent the first-line treatment. Resistant cases or those associated with frequent relapses, may benefit from immunosuppressive therapy such as azathioprine or from TNF antibodies $(2,8,12,17,21)$.

Pyoderma gangrenosum (PG). PG, considered initially an infectious disease, is a cutaneous manifestation of immune system dysregulation (12). PG is most frequently seen in association with a systemic disease, the most common of these associations being IBD (22). The prevalence of PG in IBD varies from 0.4 to $3 \%$, with some studies indicating a higher proportion in UC compared with CD $(2,9,12,17,23,24)$. Conversely, up to $50 \%$ of PG patients have underlying IBD (25). $\mathrm{PG}$ is more frequently seen in women, and it is associated with familial history of UC, colonic involvement, permanent stoma, eye involvement and EN (19). PG lesions can appear before, at the same time or after the gastrointestinal manifestations of IBD (9). 
PG may vary in clinical presentation and course. The lesions are usually preceded by a trauma, phenomenon known as pathergy. The trauma can be minimal and can precede the skin lesions with many years. The typical skin lesion starts as a sterile pustule that rapidly enlarges due to tissue necrosis; painful ulcers then develop, with undermined violaceous borders and purulent cover. The ulcerations can be solitary or multiple, unilateral or bilateral, with dimensions ranging from centimeters to an entire limb. They can expose tendons, muscles, and deep tissues. Healing is usually associated with scarring, cribriform scars being frequently seen. The lesions can affect any part of the body, but are seen most often on the lower part of the legs and adjacent to stomas $(26,27)$. Peristomal PG is rare and occurs near the site of stoma formation after surgical treatment for IBD, most likely reflecting pathergy; risk factors associated with its development are female sex, presence of other autoimmune disorders and increased body mass index (9).

Diagnosis in PG is based on the clinical picture, but investigations may be helpful to exclude other skin conditions such as infections, necrotizing vasculitis, arterial or venous ulcerations, and malignancies. There are no specific features on the histological exam, a perivascular lymphocytic infiltrate accompanied by a prominent dermal neutrophilic infiltrate being seen (27). The correlation between PG and IBD activity is controversial, as it may parallel IBD activity or run an independent course (21).

Therapy should aim at rapid healing of skin lesions because PG can be a debilitating disease. There are no data sustaining the need for different therapeutic strategies in PG associated with IBD, compared with PG associated with other diseases (21). Moreover, PG may resolve with treatment of underlying IBD (2). The mainstay of treatment is immunosuppression. Local therapy with intralesional or potent topical corticosteroids, topical calcineurin inhibitors, topical dapson can be effective in treating early and mild lesions (27). Daily wound care should be performed. In case of refractory, widespread lesions or in case of active associated IBD, systemic therapy should be started. Corticosteroids have been considered the first-line treatment, other options being oral sulfasalazine, dapsone, imunosupresive agents such as azathioprine, cyclophosphamide, cyclosporine, methotrexate, tacrolimus and mycophenolate mofetil $(2,12,21)$. Response to therapy varies, however, and refractory cases were reported. Tumor necrosis factor $\alpha$ (TNF- $\alpha$ ) inhibitors, especially Infliximab and Adalimumab, are highly effective in treating recalcitrant disease and some favor that they should be considered first-line agents in PG treatment. Infliximab should be considered if a rapid response to corticosteroids cannot be achieved, showing usually a prompt response $(2,12,21)$. Adalimumab is also effective, even in non-responsive to Infliximab patients (28). In patients with UC, complete colectomy may be needed but does not guarantee the complete resolution of $\mathrm{PG}$ lesions (12). In the particular case of peristomal PG, closure of the stoma might lead to resolution of PG lesions (21). In more than $25 \%$ of the cases, PG has a tendency to recur following successful treatment, frequently in the same place as the initial episode, and may require maintenance therapy to ensure continued remission $(21,27)$.
Pyostomatitis vegetans $(P V)$. $\mathrm{PV}$ is a rare reactive manifestation of IBD characterized by the presence of pustules, abscesses, ulcerations and vegetative plaques predominantly on the lips and oral mucosa (29). PV can be observed at any age, but its prevalence is higher in the 20-59 years age range, and it is more frequently described in men (29). All PV cases described in the literature have been associated with IBD (12). Moreover, an association with PG of the skin has been reported (12). Although the pathogenesis of IBD is poorly understood, a dysregulation of the immune system is considered the cause (29). PV lesions tend to appear months or years after IBD, but sometimes the gastrointestinal symptoms may be minimal; in these cases the diagnosis of PV should lead to a prompt referral of the patient to a bowel investigation (29).

Clinically, oral lesions appear as multiple friable pustules, with an erythematous and thickened mucosa that often ruptures leading to ulcerations and erosions. Pain and discomfort are variable, while fever and enlarged, tender lymph nodes can be present (29). Eosinophilia is present in most of the cases, and the histopathological exam reveals intraepithelial and/or subepithelial microabscesses composed of eosinophils and neutrophils. Diagnosis is based on the clinical picture, association with IBD, eosinophilia, negative cultures and histological features (29). PV tends to mirror the underlying disease activity and treatment should be focused on the treatment of IBD, essential for sustained response (9). Local treatment with antiseptic mouthwashes and topical corticosteroids can be used in order to obtain temporary relief. Effective systemic treatments include corticosteroids, TNF- $\alpha$ inhibitors, steroidsparing regimens such as dapsone or azathioprine; complete colectomy is another option (12). As in PG, recurrences after treatment are common $(9,12,29)$.

Sweet's syndrome (SS). Sweet's syndrome or acute febrile neutrophilic dermatosis is part of a group of conditions characterized by the accumulation of neutrophils in the skin. Sweet's syndrome has been rarely associated with IBD, especially with CD and less commonly with UC (30). Besides IBD, Sweet's syndrome can be associated with other systemic diseases, especially malignancies, or can be drug-related (31). SS was only recently recognized as an EIM in IBD $(32,33)$. In the context of IBD, SS appears more frequently in women, between 30 and 50 years of age, seems to be associated with colonic involvement, and other EIMs $(30,31)$. SS parallels IBD activity in the majority of cases, but may precede the diagnosis of IBD or can occur after colectomy (34). The pathogenesis of SS is unclear, type III hypersensitivity, T-lymphocyte dysfunction, or an association with histocompatibility antigens has been suggested as potential mechanisms (21).

Clinically SS is characterized by the acute onset of succulent, tender, red to purple, inflammatory nodules, papules and plaques that can become confluent, accompanied by burning or pain, usually affecting the upper limbs, face and neck. Lesions can be preceded by trauma (pathergy), similar to PG. Skin lesions are typically associated with fever; other symptoms include arthralgias, headache, and fatigue (31). SS can affect other organs besides the skin, including lungs, muscles, joints, liver, kidney and eyes. Leukocytosis and neutrophilia are frequently seen and the histopathological exam reveals 
the presence of papillary edema, with a dense neutrophilic infiltrate and leukocytoclasia (31).

Systemic corticosteroids are first-line treatment and usually lead to rapid resolution of the lesions. Immunosuppressive therapy or biological agents should be considered in resistant or highly relapsing cases (21). Of note, azathioprine has been implicated in the development of SS in some IBD patients $(35,36)$.

Oral aphthous ulcers. Oral aphthous ulcers or aphthae represent the most common oral mucosal finding in IBD patients (9). Aphthae are present in up to $20 \%$ of the normal population and in up to one-third of IBD patients (9). The clinical picture is the same, consisting of multiple shallow, round ulcers surrounded by an erythematous 'halo' and covered by a fibrinous exudate. In the presence of aphthae, IBD must be suspected even if intestinal symptoms are not yet present $(9,12,13)$. Increased IBD activity is considered a trigger factor for oral lesions, together with stress, food or drug allergies, trauma and nutritional deficiencies $(9,12)$. Diagnosis is based on the clinical picture, although in case of big, treatment resistant ulcer, especially in smokers a biopsy is needed in order to rule out a squamous cell carcinoma. Treatment consists in addressing the underlying cause and local symptomatic therapy with anesthetics and topical corticosteroids $(9,12,13)$.

Cutaneous polyarteritis nodosa $(C P N)$. $\mathrm{CPN}$ is a rare cutaneous EIMs of IBD, defined as a chronic, recurring vasculitis of the small and medium sized arteries, localized in the reticular dermis and subcutaneous tissue. Approximately $10 \%$ of the CPN cases are associated with IBD, and the diagnosis of CPN can precede occasionally the gastrointestinal symptoms (12). The pathogenesis is uncertain, but an immune complex etiology is suspected (37). Clinically, CPN presents as a tender nodule, located on the lower extremity, mimicking EN, PG, and metastatic CD $(12,38)$. The diagnosis is based on the histopathological exam which shows a localized perivascular infiltrate, with the absence of the inflammation in the surrounding tissue (12). CPN has an independent course of the underlying IBD and treatment is based on low-dose corticosteroids and NSAINs $(9,12)$.

Necrotising vasculitis $(N V)$. Necrotising vasculitis defines a group of diseases characterized by inflammatory lesions of the blood vessels and necrosis of the vessel wall, and is now a recognized feature of a broad range of diseases with different etiopathogenesis (39). NV has been reported in association with $\operatorname{IBD}(9,12,40-42)$. Clinically, palpable purpura, which progresses to ulceration and even gangrene, located typically on the legs, is seen. The histopathological exam reveals a neutrophilic infiltrate and nuclear debris in the vessel wall of the postcapillary venules, distinguishing the disease from PG and CPN (39). Usually NV appears after a diagnosis of IBD, although cases with prior development have been reported (40-42). Systemic corticosteroids are the first line treatment, leading in most cases to the resolution of skin lesions (39).

Thrombosis. IBD confers an increased risk of thrombosis, which can manifest as deep venous thrombosis or thromboembolic disease in the dermatologic patient (43). A meta-analysis conducted by Nguyen et al (44) showed that IBD patients have a 2.8-fold increased relative risk of a first episode of venous thromboembolism, the risk being similar for CD and UC. Increased risk is correlated directly with disease activity, although thrombosis can occur also during remission (45). Intestinal inflammation leads to the release of inflammatory cytokines and consequently to the development of a prothrombotic state (43). Thrombosis can manifest in atypical locations, such as the axillary and subclavian venous system and appears in younger age group compared with the general population (46). Prevention and treatment are challenging and have to consider the episodic nature of IBD and the high risk of gastrointestinal bleeding in active disease. Use of anticoagulant thromboprophylaxis with low molecular weight heparin, unfractionated heparin, or fondaparinux is recommended for hospitalized patients with IBD in remission or with active flares of IBD without major gastrointestinal bleeding, including patients with minor gastrointestinal bleeding, although the last is often considered an absolute contraindication to anticoagulant therapy (43). For patients with major gastrointestinal bleeding, mechanical prophylaxis is recommended (44). In outpatients with active IBD without previous history of thrombosis, anticoagulant prophylaxis is not recommended, in contrast to those with positive history in which prophylaxis is recommended during flares $(43,44)$.

Epidermolysis bullosa acquisita (EBA). EBA is a rare autoimmune blistering disease caused by IgG anutoantibodies directed against collagen VII, characterized by subepidermal blisters that progress often to scarring in anatomic areas prone to trauma, such as hands and feet (47). EBA has been associated with IBD, collagen VII being the shared antigen. CD has been described in approximately $30 \%$ of EBA patients, while UC is reported with a lower frequency (47). EBA is more frequently associated with CD because of the higher incidence of type VII collagen autoimmunity in CD, compared with UC (47). In the majority of cases, the onset of the gastrointestinal symptoms proceeds by years or occurs simultaneously with the skin disease, although mild gastrointestinal symptoms can be overlooked (47). A variety of treatments have been tried for EBA, including dapsone, sulfapyridine, and colchicine in mild forms, and cyclosporine, mycophenolate mofetil, rituximab, intravenous immunoglobulins and extracorporeal photochemotherapy in resistant and severe forms (9).

\section{Associate cutaneous manifestations}

As mentioned before, associated conditions are more frequently observed in the context of IBD, without sharing the pathogenetic mechanism or the histopathological findings with the underlying disease $(8,9)$. They are likely related to HLA linkage and the chronic inflammatory nature of IBD (17). Psoriasis, vitiligo, eczema, clubbing of the nails and acrodermatitis enteropatica were all included in this category of EIMs (17). Recently, Kim et al (48) conducted a study in order to clarify the association between IBD and inflammatory skin diseases and found that rosacea, psoriasis and atopic dermatitis were significantly associated with IBD, whereas the association between IBD and autoimmune skin diseases including vitiligo and alopecia areata were less marked or nonexistent. 


\section{Drug-related cutaneous manifestations}

Treatment of IBD, as in other inflammatory diseases, implies a balance between controlling active disease and complications on one hand and side effects of medication on the other hand (49). Most of the medications used in IBD supress the immune system and are associated with multiple side effects. Gold standard of treatment is represented by systemic corticosteroids, which remain the first line option for the induction of remission in active disease (49). 5-Aminosalicylate medications are widely used, but they are often not sufficient to maintain long-term remission (49). Immune-modulating therapies such as 6-mercaptopurine and methotrexate are used to maintain remission and to prevent immunogenicity to biologic agents. 6-mercaptopurine is associated with alopecia, skin rashes, Sweet syndrome, and skin cancer. Cutaneous and mucosal side effects associated with methotrexate include oral ulcerations, skin ulcerations, mild alopecia, acral erythema, epidermal necrosis, and vasculitis.

In recent years, the use of monoclonal antibody-based or biologic therapy has increased dramatically in the treatment of moderate to severe IBD, both for induction and maintenance of remission in patients with active disease (49). Anti-TNF agents, such as infliximab, adalimumab, golimumab, and certolizumab can be used in IBD. Approximately 25\% of the patients treated with anti-TNF agents develop cutaneous side effects, typically months to years after the initiation of the treatment, although some cutaneous reactions can occur as early as one week after the initiation of the therapy (50). It has been shown that smoking, female sex, and CD are risk factors for anti-TNF-induced cutaneous lesions (50). Fortunately, with adequate dermatological treatment the discontinuation of anti-TNF is only rarely required (50). However, interruption is recommended when unbearable reactions persist despite optimal treatment. Worth mentioning is that some reactions need several months to subside after the interruption of the offending drug (51). Taking into account that skin reactions are a consequence of the inhibition of the TNF function in the skin, being class-specific, switching between different anti-TNF antibodies is not a very useful strategy. However, biologic agents from other classes can be an option. Ustekinumab exhibits therapeutic effects on both Th17 axis and psoriasis, it is not associated with xerosis, eczema, psoriasis or palmoplantar pustulosis, and can induce regrowth in patients with extensive alopecia areata (50-52). Vedolizumab could be an option, but exhibits a gut-specific mechanism of action, and has no effects on EIMs (53).

Segaert and Hermans (50) tried to classify anti-TNF skin reactions into the following: xerosis, eczema, psoriasiform dermatitis, psoriasis, palmo-plantar pustulosis, infections, skin cancer, alopecia and others.

Xerosis. Xerosis or dry skin was observed in more than $40 \%$ of patients treated with anti-TNF agents, being more frequent in those with atopic background, in older age or during winter season $(50,51)$. Xerosis is frequently accompanied by eczema or psoriasiform eczema (51). Prevention of xerosis implies avoidance of drying skin care products, frequent bathing, and very hot water, and switching to cleansing oils and lukewarm water. Emollients should be used daily, especially in patients with a history of atopic dermatitis or dry skin $(50,51)$.
Eczema/psoriasiform eczema. Cleynen et al (51) observed eczema or dermatitis in $23.5 \%$ of IBD patients receiving infliximab, while Esmailzadeh et al (54), reported it in $16.3 \%$ of infliximab-treated patients without cutaneous psoriasis. Moreover, the latter identified a personal history of atopy as a predictive factor for the occurrence of eczema (54). Clinically, poorly defined, scally, pruritic macules or plaques develop, usually with topography reminiscent of atopic dermatitis. Histology shows typical features for eczema, including spongiosis, exocytosis, dermal perivascular infiltration, ruling out psoriasis $(51,55)$. At the same time, aggravation of a previous dermatitis, namely stasis or contact dermatitis, was observed in patients treated with anti-TNF agents $(50,56)$.

Psoriasiform eczema or psoriasiform dermatitis was the most frequently encountered skin reaction by Cleynen et al (51), being described in $30.6 \%$ of the cases. Psoriasiform lesions present as scaly, erythematous plaques, with an orange-red hue, resembling psoriatic lesions. Psoriasiform lesions are usually located on the scalp and flexural regions mimicking inverse psoriasis, but can also be seen on the face and trunk. Nail involvement is rare (3.3\%), and histology shows features of eczema $(8,50)$. The distinction between psoriasiform dermatitis and psoriasis can be clinically challenging. The lack of sharp demarcation, the absence of silvery-white scales, the frequent bacterial superinfection and the rapid response to corticosteroids favour the diagnosis of eczema, and histopathological examination can be performed in doubtful cases (50).

Bacterial superinfection of is a common feature, both of eczema and psoriasiform eczema, leading to oozing or crusted lesions. Differential diagnosis includes skin infections, allergic reactions, and IBD-associated skin lesions $(8,50)$.

Eczema lesions can usually be controlled with topical treatment, without withdrawal of the medication. Topical treatment options include corticosteroids, vitamin D analogues, and ultraviolet therapy, besides the general methods used to prevent xerosis. In case of bacterial infection, a combination of corticosteroid with an antibiotic or a course of oral antibiotics can be used $(8,50)$.

Psoriasis. Psoriasis, characterised by sharply defined orangered or deep red plaques, covered by silvery-white scales, is rarely described in IBD patients receiving anti-TNF therapy (51). Superinfection is usually absent, and pruritus may or may not be present. Response to therapy is slower than in psoriasiform eczema. Some patients show both psoriasiform dermatitis and psoriasis at the same time. Guttate and pustular psoriasis have also been described (50). A topical combination of a vitamin D analogue and a potent corticosteroid is usually enough to control the disease, but acitretin, methotrexate, or cyclosporine can be used for resistant cases (50).

Palmoplantar pustulosis. Palmoplantar pustulosis, characterised by recurrent crops of sterile pustules evolving into erythematosquamous plaques, is one of the first described skin reaction associated with anti-TNF agents (50). The reaction seems to be related to smoking, but to a lesser extent than the sporadic form. Palmoplantar pustulosis can appear alone, or can be accompanied by chronic plaque type, guttate, or pustular psoriasis of the body or by psoriasiform eczema (50). Smoking cessation, as well as an ultrapotent corticosteroid 
combined with salicylic acid or coal tar is prescribed. Acitretin, methotrexate, or oral corticosteroids are used for recalcitrant cases (50).

Skin infections. Infections are the most frequently encountered cutaneous side effect of anti-TNF agents, with a frequency ranging from 12.5 to $57.1 \%$ of patients with cutaneous adverse events (50). Bacterial infections were described most frequently, but viral, fungal and even opportunistic infections can be present (50).

Skin cancer. Some studies indicate a moderately increased risk for non-melanoma and melanoma skin cancer in pacients receiving anti-TNF agents (50). Moreover, some melanoma patients experienced explosive metastasis following the initiation of anti-TNF agents (57).

Alopecia. Patients treated with anti-TNF agents can develop both alopecia associated with highly inflammatory psoriasiform dermatitis and alopecia areata. Alopecia areata occurs on non-inflamed skin, usually as patchy alopecia areata, but alopecia areata totalis and universalis were also described. Exacerbation of known alopecia areata, as well as new onset of the disease has been reported (50). An ultrapotent topical corticosteroid can be used in patchy alopecia areata, while in rapidly progressive alopecia, alopecia totalis or universalis, a switch to ustekinumab or vedolizumab should be considered (50).

Other skin reactions. Rare skin reactions associated with anti-TNF treatment include injection site reactions and infusion reactions, cutaneous lupus, lichen planus, vasculitis, granulomatous reactions, acne, rosacea, amicrobial pustulosis, cutaneous lymphoma, and compromised wound healing.

Discontinuation of the causal anti-TNF agent due to cutaneous side effects is recommended only when unbearable cutaneous reactions persist despite optimal dermatological care. Taking into account that cutaneous adverse events are a consequence of the inhibition of the physiological function of TNF in the skin, switching between different anti-TNF antibodies is not very useful.

\section{Conclusions}

Cutaneous manifestations in IBD are frequent and comprise a broad spectrum of diseases, ranging from mild to severe and sometimes debilitating lesions. Some of the cutaneous manifestations can be the presenting sign of an underlying intestinal disease, leading to the screening for non-detected IBD even in the absence of symptoms. Cutaneous EIMs are divided into 4 categories: i) disease-specific lesions that show the same histopathologic findings as the underlying gastrointestinal disease, ii) reactive lesions which are inflammatory lesions that share a common pathogenetic mechanism but do not share the same pathology with the gastrointestinal disease and iii) associated conditions are more frequently observed in the context of IBD, without sharing the pathogenetic mechanism or the histopathological findings with the underlying disease $(8,9)$ and iv) drug-related skin reactions. Treatment can be challenging and paradoxical skin reactions may occur in response to anti-TNF treatment, leading to a fourth category of skin manifestations, namely drug-related skin reactions.

\section{Acknowledgements}

Not applicable.

\section{Funding}

No funding was received.

\section{Availability of data and materials}

Not applicable.

\section{Author's contribution}

MAB and AFV collected the literature. LU, RC, SCS and IC were responsible for the literature review, manuscript drafting and critical revision of the manuscript for important intellectual content. All authors read and approved the manuscript.

\section{Ethics approval and consent to participate}

Not applicable.

\section{Patient consent for publication}

Not applicable

\section{Competing interests}

The authors declare that they have no competing interests.

\section{References}

1. Tontini GE, Vecchi M, Pastorelli L, Neurath MF and Neumann H: Differential diagnosis in inflammatory bowel disease colitis: State of the art and future perspectives. World J Gastroenterol 21: 21-46, 2015.

2. Vavricka SR, Schoepfer A, Scharl M, Lakatos PL, Navarini A and Rogler G: Extraintestinal manifestations of inflammatory bowel disease. Inflamm Bowel Dis 21: 1982-1992, 2015.

3. Vavricka SR, Rogler G, Gantenbein C, Spoerri M, Prinz Vavricka M, Navarini AA, French LE, Safroneeva E, Fournier N, Straumann A, et al: Chronological order of appearance of extraintestinal manifestations relative to the time of IBD diagnosis in the Swiss Inflammatory Bowel Disease Cohort. Inflamm Bowel Dis 21: 1794-1800, 2015.

4. Monsén U, Sorstad J, Hellers G and Johansson C: Extracolonic diagnoses in ulcerative colitis: An epidemiological study. Am J Gastroenterol 85: 711-716, 1990.

5. Das KM: Relationship of extraintestinal involvements in inflammatory bowel disease: New insights into autoimmune pathogenesis. Dig Dis Sci 44: 1-13, 1999.

6. Vavricka SR, Brun L, Ballabeni P, Pittet V, Prinz Vavricka BM, Zeitz J, Rogler G and Schoepfer AM: Frequency and risk factors for extraintestinal manifestations in the Swiss inflammatory bowel disease cohort. Am J Gastroenterol 106: 110-119, 2011

7. Bernstein CN, Blanchard JF, Rawsthorne $\mathrm{P}$ and Yu N: The prevalence of extraintestinal diseases in inflammatory bowel disease: A population-based study. Am J Gastroenterol 96: 1116-1122, 2001.

8. Greuter T, Navarini A and Vavricka SR: Skin manifestations of inflammatory bowel disease. Clin Rev Allergy Immunol 53: 413-427, 2017.

9. Hagen JW, Swoger JM and Grandinetti LM: Cutaneous manifestations of Crohn disease. Dermatol Clin 33: 417-431, 2015. 
10. Xavier RJ and Podolsky DK: Unravelling the pathogenesis of inflammatory bowel disease. Nature 448: 427-434, 2007.

11. Rankin GB, Watts HD, Melnyk CS and Kelley ML Jr: National Cooperative Crohn's Disease Study: Extraintestinal manifestations and perianal complications. Gastroenterology 77: 914-920, 1979

12. Trash B, Patel M, Shah KR, Boland CR and Menter A: Cutaneous manifestations of gastrointestinal disease. Part II. J Am Acad Dermatol 68: 211.e1-33, 2013.

13. Muhvić-Urek M, Tomac-Stojmenović $M$ and MijandrušićSinčić B: Oral pathology in inflammatory bowel disease. World J Gastroenterol 22: 5655-5667, 2016.

14. Lebwohl M, Fleischmajer R, Janowitz H, Present D and Prioleau PG: Metastatic Crohn's disease. J Am Acad Dermatol 10: 33-38, 1984.

15. Hawryluk EB, Izikson L and English JC III: Non-infectious granulomatous diseases of the skin and their associated systemic diseases: An evidence-based update to important clinical questions. Am J Clin Dermatol 11: 171-181, 2010.

16. Guest GD and Fink RL: Metastatic Crohn's disease: Case report of an unusual variant and review of the literature. Dis Colon Rectum 43: 1764-1766, 2000.

17. Rahvar M and Kerstetter J: Cutaneous manifestation of gastrointestinal disease. J Gastrointest Oncol 7 (Suppl 1): S44-S54, 2016

18. Chowaniec M, Starba A and Wiland P: Erythema nodosum review of the literature. Reumatologia 54: 79-82, 2016.

19. Farhi D, Cosnes J, Zizi N, Chosidow O, Seksik P, Beaugerie L, Aractingi S and Khosrotehrani K: Significance of erythema nodosum and pyoderma gangrenosum in inflammatory bowel diseases: A cohort study of 2402 patients. Medicine (Baltimore) 87: 281-293, 2008

20. Apgar JT: Newer aspects of inflammatory bowel disease and its cutaneous manifestations: A selective review. Semin Dermatol 10: $138-147,1991$

21. Harbord M,Annese V,VavrickaSR,Allez M,Barreiro-de Acosta M, Boberg KM, Burisch J, De Vos M, De Vries AM, Dick AD, et al European Crohn's and Colitis Organisation: The first European evidence-based consensus on extra-intestinal manifestations in inflammatory bowel disease. J Crohn's Colitis 10: 239-254, 2016.

22. Callen JP: Pyoderma gangrenosum. Lancet 351: 581-585, 1998.

23. Menachem Y and Gotsman I: Clinical manifestations of pyoderma gangrenosum associated with inflammatory bowel disease. Isr Med Assoc J 6: 88-90, 2004.

24. Polcz M, Gu J and Florin T: Pyoderma gangrenosum in inflammatory bowel disease: The experience at Mater Health Services' Adult Hospital 1998-2009. J Crohn's Colitis 5: 148-151, 2011.

25. Rothfuss KS, Stange EF and Herrlinger KR: Extraintestinal manifestations and complications in inflammatory bowel diseases. World J Gastroenterol 12: 4819-4831, 2006.

26. Cosgarea R, Senilă SC, Badea R and Ungureanu L: Pyoderma gangrenosum with spleen involvement. Review of the literature and case report. J Dermatol Case Rep 10: 26-31, 2016.

27. Wollina U: Pyoderma gangrenosum - a systemic disease? Clin Dermatol 33: 527-530, 2015.

28. Fonder MA, Cummins DL, Ehst BD, Anhalt GJ and Meyerle JH: Adalimumab therapy for recalcitrant pyoderma gangrenosum. $\mathrm{J}$ Burns Wounds 5: e8, 2006.

29. Femiano F, Lanza A, Buonaiuto C, Perillo L, Dell'Ermo A and Cirillo N: Pyostomatitis vegetans: A review of the literature. Med Oral Patol Oral Cir Bucal 14: E114-E117, 2009.

30. Hindryckx P, Novak G, Costanzo A and Danese S: Diseaserelated and drug-induced skin manifestations in inflammatory bowel disease. Expert Rev Gastroenterol Hepatol 11: 203-214, 2017.

31. Marzano AV, Borghi A, Wallach D and Cugno M: A comprehensive review of neutrophilic diseases. Clin Rev Allergy Immunol 54: 114-130, 2018.

32. Travis S, Innes N, Davies MG, Daneshmend T and Hughes S; The South West Gastroenterology Group: Sweet's syndrome: An unusual cutaneous feature of Crohn's disease or ulcerative colitis Eur J Gastroenterol Hepatol 9: 715-720, 1997.

33. Ytting H, Vind I, Bang D and Munkholm P: Sweet's syndrome an extraintestinal manifestation in inflammatory bowel disease. Digestion 72: 195-200, 2005

34. Ardizzone S, Puttini PS, Cassinotti A and Porro GB: Extraintestinal manifestations of inflammatory bowel disease. Dig Liver Dis 40 (Suppl 2): S253-S259, 2008.

35. El-Azhary RA, Brunner KL and Gibson LE: Sweet syndrome as a manifestation of azathioprine hypersensitivity. Mayo Clin Proc 83: 1026-1030, 2008.
36. Choonhakarn $\mathrm{C}$ and Chaowattanapanit S: Azathioprine-induced Sweet's syndrome and published work review. J Dermatol 40: 267-271, 2013

37. Diaz-Perez JL, Schroeter AL and Winkelmann RK: Cutaneous periarteritis nodosa: Immunofluorescence studies. Arch Dermatol 116: 56-58, 1980 .

38. Borrie P: Cutaneous polyarteritis nodosa. Br J Dermatol 87: 87-95, 1972

39. Ozen S: The changing face of polyarteritis nodosa and necrotizing vasculitis. Nat Rev Rheumatol 13: 381-386, 2017.

40. Akbulut S, Ozaslan E, Topal F, Albayrak L, Kayhan B and Efe C: Ulcerative colitis presenting as leukocytoclastic vasculitis of skin. World J Gastroenterol 14: 2448-2450, 2008.

41. Zlatanic J, Fleisher M, Sasson M, Kim P and Korelitz BI: Crohn's disease and acute leukocytoclastic vasculitis of skin. Am J Gastroenterol 91: 2410-2413, 1996.

42. Iannone F, Scioscia C, Musio A, Piscitelli D and Lapadula G: Leucocytoclastic vasculitis as onset symptom of ulcerative colitis. Ann Rheum Dis 62: 785-786, 2003.

43. Lentz SR: Thrombosis in the setting of obesity or inflammatory bowel disease. Blood 128: 2388-2394, 2016

44. Nguyen GC, Bernstein CN, Bitton A, Chan AK, Griffiths AM, Leontiadis GI, Geerts W, Bressler B, Butzner JD, Carrier M, et al: Consensus statements on the risk, prevention, and treatment of venous thromboembolism in inflammatory bowel disease: Canadian Association of Gastroenterology. Gastroenterology 146: 835-848.e6, 2014

45. Grainge MJ, West J and Card TR: Venous thromboembolism during active disease and remission in inflammatory bowel disease: A cohort study. Lancet 375: 657-663, 2010.

46. Zitomersky NL, Verhave M and Trenor CC III: Thrombosis and inflammatory bowel disease: A call for improved awareness and prevention. Inflamm Bowel Dis 17: 458-470, 2011.

47. Reddy H, Shipman AR and Wojnarowska F: Epidermolysis bullosa acquisita and inflammatory bowel disease: A review of the literature. Clin Exp Dermatol 38: 225-229, 2013.

48. Kim M, Choi KH, Hwang SW, Lee YB, Park HJ and Bae JM: Inflammatory bowel disease is associated with an increased risk of inflammatory skin diseases: A population-based crosssectional study. J Am Acad Dermatol 76: 40-48, 2017.

49. Shapiro JM, Subedi S and LeLeiko NS: Inflammatory bowel disease. Pediatr Rev 37: 337-347, 2016.

50. Segaert $S$ and Hermans C: Clinical signs, pathophysiology and management of cutaneous side effects of anti-tumor necrosis factor agents. Am J Clin Dermatol 18: 771-787, 2017.

51. Cleynen I, Van Moerkercke W, Billiet T, Vandecandelaere P, Vande Casteele N, Breynaert C, Ballet V, Ferrante M, Noman M, Assche GV, et al: Characteristics of skin lesions associated with anti-tumor necrosis factor therapy in patients with inflammatory bowel disease: A cohort study. Ann Intern Med 164: 10-22, 2016.

52. Guttman-Yassky E, Ungar B, Noda S, Suprun M, Shroff A, Dutt R, Khattri S, Min M, Mansouri Y, Zheng X, et al: Extensive alopecia areata is reversed by IL-12/IL-23p40 cytokine antagonism. J Allergy Clin Immunol 137: 301-304, 2016.

53. Peyrin-Biroulet L, Van Assche G, Gómez-Ulloa D, GarcíaÁlvarez L, Lara N, Black CM and Kachroo S: Systematic review of tumor necrosis factor antagonists in extraintestinal manifestations in inflammatory bowel disease. Clin Gastroenterol Hepatol 15: 25-36.e27, 2017.

54. Esmailzadeh A, Yousefi P, Farhi D, Bachmeyer C, Cosnes J, Berenbaum F, Duriez P, Aractingi S and Khosrotehrani K: Predictive factors of eczema-like eruptions among patients without cutaneous psoriasis receiving infliximab: A cohort study of 92 patients. Dermatology 219: 263-267, 2009.

55. Lee HH, Song IH, Friedrich M, Gauliard A, Detert J, Röwert J, Audring H, Kary S, Burmester GR, Sterry W, et al: Cutaneous side-effects in patients with rheumatic diseases during application of tumour necrosis factor-alpha antagonists. Br J Dermatol 156: 486-491, 2007.

56. Flendrie M, Vissers WH, Creemers MC, de Jong EM, van de Kerkhof PC and van Riel PL: Dermatological conditions during TNF-alpha-blocking therapy in patients with rheumatoid arthritis: a prospective study. Arthritis Res Ther 7: R666-R676, 2005.

57. Fulchiero GJ Jr, Salvaggio H, Drabick JJ, Staveley-O'Carroll K, Billingsley EM, Marks JG and Helm KF: Eruptive latent metastatic melanomas after initiation of antitumor necrosis factor therapies. J Am Acad Dermatol 56 (Suppl 5): S65-S67, 2007.

This work is licensed under a Creative Commons Attribution-NonCommercial-NoDerivatives 4.0 International (CC BY-NC-ND 4.0) License. 